\title{
Hemithyroidectomy for low-risk follicular carcinoma of the thyroid: results from a regional hospital
}

\author{
Tam-Lin $\underline{C h o w}^{1}$, frCs, Susanna Wai-Yin $\underline{\text { Tam }}^{1}$, frCs, Chi-Yee $\underline{C h o i}^{1}$, frCs, Wilson Wai-Yin $\underline{K w a n}^{1}$, frCs
}

INTRODUCTION Excellent outcomes after conservative thyroid surgery for low-risk follicular thyroid carcinoma (FTC) have been reported from highly specialised centres. However, it is uncertain whether low-volume hospitals can achieve similar treatment results.

METHODS At our institution, 49 patients with FTC were treated during the period 1991-2014. Patients with minimally invasive FTC (MIFTC) were usually treated with hemithyroidectomy. The demographic data, pathology, treatment modality and oncological outcomes of these patients were retrospectively evaluated.

RESULTS The tumours were classified as Stage I in $40.8 \%$ of patients, Stage II in $32.7 \%$, Stage III in $20.4 \%$ and Stage IV in $6.1 \%$, according to the TNM classification system. Only $4(8.2 \%)$ patients had widely invasive FTC (WIFTC). Vascular invasion or capsular invasion alone occurred in $9(19.1 \%)$ and $19(40.4 \%)$ patients, respectively, while 19 (40.4\%) patients had simultaneous vascular and capsular invasions. 34 (69.4\%) patients with MIFTC initially underwent hemithyroidectomy, while $15(30.6 \%)$ patients underwent total thyroidectomy. Ten patients who underwent total thyroidectomy received radioactive iodine ablation. The mean follow-up duration was $86.9 \pm 56.6$ months. There was no disease-specific mortality, although two patients with WIFTC remained alive with disease at the end of the study. The five-, ten- and 15-year overall survival rates were $95 \%, 91 \%$ and $84 \%$, respectively. Five patients from the hemithyroidectomy group died due to other illnesses with no evidence of FTC.

CONCLUSION Satisfactory disease control and excellent survival for MIFTC is achievable by hemithyroidectomy in community hospitals. Total thyroidectomy should be reserved for WIFTC or aggressive tumours with nodal or distant metastasis.

Keywords: follicular thyroid carcinoma, Hürthle cell, neoplasm metastasis, prognosis, thyroidectomy

\section{INTRODUCTION}

Although the incidence of thyroid carcinoma is rising worldwide, the frequency of follicular thyroid carcinoma (FTC) remains static $^{(1)}$ and comprises only $10 \%$ of all thyroid cancers. ${ }^{(1)}$ Its rarity, together with a markedly variable clinical course, renders the best treatment elusive. The procedure of choice can be hemithyroidectomy or total thyroidectomy. It should also be noted that while deciding on the best treatment for patients with FTC, the oncological outcome should be balanced against the increased morbidity associated with more extensive surgery for FTC.

FTC can be classified as minimally invasive FTC (MIFTC) or widely invasive FTC (WIFTC) based on histopathological features. ${ }^{(2)}$ MIFTC is characterised by foci of small-to-medium capsular vessel invasion or up to full-thickness capsular transgression by the tumour, but not beyond. Extensive vascular invasion of the tumour capsule is considered to be WIFTC. MIFTC is generally reckoned to be less aggressive, with a better prognosis than WIFTC. ${ }^{(3,4)}$ Therefore, hemithyroidectomy has been proposed as an adequate operation for MIFTC, while total thyroidectomy plus adjuvant radioactive iodine (RAI) are reserved for WIFTC. Excellent oncological outcomes after conservative surgery for low-risk FTC have been reported from high-volume centres. ${ }^{(5,6)}$ However, to the best of our knowledge, the treatment results of conservative thyroid surgery for MIFTC in regional hospitals with lower caseloads have hardly ever been reported in the literature. It is uncertain whether the good results seen in specialised centres can be comparably achieved in hospitals with low patient volumes.

In this study, we present the treatment outcome for FTC at our hospital, which is a low-volume institution vis-à-vis the caseload of patients with FTC, with a focus on whether hemithyroidectomy without RAI was appropriate for MIFTC.

\section{METHODS}

This was a retrospective survey of the treatment outcomes of patients with FTC whose information was prospectively collected in the cancer registry at the Department of Surgery, United Christian Hospital, Kowloon, Hong Kong, from May 1991 to November 2014. All of the retrieved patients had histopathologically verified FTC, with unequivocal capsular or vascular invasion by tumour cells. Hürthle cell carcinoma, an oncocytic variant of FTC, was included in this cohort, as it has been classified within the FTC category based on the World Health Organization system. ${ }^{(2)}$ The demographic data, types of surgery, pathology results, adjuvant RAI and oncological outcomes were extracted and evaluated from the registry and patient records.

Our institution is a community hospital with 184 adult surgical beds, serving a population of 600,000 in the East Kowloon region of Hong Kong. Between April 2013 and March

${ }^{1}$ Department of Surgery, United Christian Hospital, Kowloon, Hong Kong

Correspondence: Dr Tam-Lin Chow, Consultant, Department of Surgery, United Christian Hospital, 130 Hip Wo Street, Kwun Tong, Kowloon, Hong Kong. tamlinc@yahoo.com 
2014, a total of 112 thyroid operations were performed at our centre, which gives an indication of its annual thyroidectomy volume. The management of differentiated thyroid carcinoma at our hospital is individualised: hemithyroidectomy is indicated for low-risk papillary carcinoma, while total thyroidectomy is prescribed for high-risk patients. With this management regime, satisfactory tumour control with low complication rates has been reported at our centre. ${ }^{(7)}$ It was also previously reported that for T1/T2 N0M0 differentiated thyroid cancer, according to the TNM Classification of Malignant Tumours (TNM), the histopathology types (i.e. papillary carcinoma, follicular carcinoma or Hürthle cell carcinoma) were found to have similar survival rates regardless of whether patients were treated with hemithyroidectomy or total thyroidectomy. ${ }^{(6)}$ Accordingly, the therapy for FTC at our hospital has evolved over the past 20 years to involve hemithyroidectomy for patients with MIFTC and total thyroidectomy followed by RAI for those with WIFTC.

During the thyroidectomy procedure, when gross invasion to adjacent structures (e.g. strap muscles or recurrent laryngeal nerve) was noticed intraoperatively, the lesion was removed en bloc with the thyroid gland for patients with WIFTC. By contrast, gross invasion to adjacent structures by MIFTC was so rare that radical resection was not considered necessary. The central compartment lymph node was normally not dissected, as is the usual practice for papillary carcinoma at our institution, ${ }^{(8)}$ due to the low risk of nodal spread for FTC. $(4,9,10)$

After surgery with or without RAI, thyroxine suppression therapy was administered to patients, who were then followed up at outpatient clinics once every three months during the first postoperative year and every six months for four years thereafter. Eventually, patients would be followed up annually. The thyroxine suppression therapy at our centre aimed to keep the level of serum thyroid-stimulating hormone below $0.27 \mathrm{mIU} / \mathrm{L}$, regardless of the degree of invasiveness of the carcinoma or the extent of thyroidectomy. Tumour surveillance was monitored through clinical examination, office ultrasonography of the neck and serum thyroglobulin (only for patients undergoing total thyroidectomy). The follow-up duration was calculated from the date of the diagnosis.

Patients were evaluated by intention-to-treat analysis; patients who underwent completion thyroidectomy six months or more after the initial hemithyroidectomy, for any reason, were considered to be hemithyroidectomy patients. By contrast, patients who underwent completion thyroidectomy within six months of the initial hemithyroidectomy were considered to be total thyroidectomy patients. Continuous variables were expressed as mean \pm standard deviation. Categorical variables were expressed as frequency and proportion. Patient survival was presented using the Kaplan-Meier curve. Data was analysed using IBM SPSS Statistics version 20 (IBM Corp, Armonk, NY, USA).

\section{RESULTS}

A total of 49 consecutive patients who fulfilled the inclusion criteria were recruited. The demographic and clinicopathological data of the patients is presented in
Table I. Demographic and clinicopathological characteristics of patients with FTC $(n=49)$.

\begin{tabular}{|c|c|}
\hline Variable & No. (\%) \\
\hline Age (yr)* & $49.7 \pm 16.2$ \\
\hline \multicolumn{2}{|l|}{ Gender } \\
\hline Female & $40(81.6)$ \\
\hline Male & $9(18.4)$ \\
\hline \multicolumn{2}{|l|}{ Tumour type } \\
\hline Follicular carcinoma & $36(73.5)$ \\
\hline Hürthle cell carcinoma & $13(26.5)$ \\
\hline Tumour size $(\mathrm{mm})^{*}$ & $36.0 \pm 19.3$ \\
\hline \multicolumn{2}{|l|}{ Degree of invasion } \\
\hline MIFTC & $45(91.8)$ \\
\hline WIFTC & $4(8.2)$ \\
\hline \multicolumn{2}{|l|}{ Invasion type $(n=47)$} \\
\hline Vascular only & $9(19.1)$ \\
\hline Capsular only & $19(40.4)$ \\
\hline Simultaneous vascular and capsular & $19(40.4)$ \\
\hline \multicolumn{2}{|l|}{ Operation } \\
\hline Hemithyroidectomy & $34(69.4)$ \\
\hline Total thyroidectomy & $15(30.6)$ \\
\hline \multicolumn{2}{|l|}{ Tumour stage ${ }^{\dagger}$} \\
\hline I & $20(40.8)$ \\
\hline II & $16(32.7)$ \\
\hline III & $10(20.4)$ \\
\hline IV & $3(6.1)$ \\
\hline \multicolumn{2}{|l|}{ Radioactive iodine therapy } \\
\hline Yes & $11(22.4)$ \\
\hline No & $38(77.6)$ \\
\hline Follow-up duration (mth)* & $86.9 \pm 56.6$ \\
\hline
\end{tabular}

*Data presented as mean \pm standard deviation. + According to TNM Classification of Malignant Tumours. FTC: follicular thyroid carcinoma; MIFTC: minimally invasive FTC; WIFTC: widely invasive FTC

Table I. The median age of the patients was $49.7 \pm 16.2$ years and the majority were female $(n=40,81.6 \%)$. There were $36(73.5 \%)$ follicular carcinomas and $13(26.5 \%)$ Hürthle cell carcinomas. Of these 49 tumours, 4 (8.2\%) were WIFTC based on histopathology. The mean tumour size was 36.0 $\pm 19.3 \mathrm{~mm}$. The majority $(\mathrm{n}=34,69.4 \%$ ) of the patients underwent hemithyroidectomy, while $15(30.6 \%)$ patients received total thyroidectomy. A total of 11 (22.4\%) patients received $\mathrm{RAI}$ - ten from the total thyroidectomy group and one from the hemithyroidectomy group.

All four patients with WIFTC in our study underwent total thyroidectomy and subsequent RAI (Fig. 1). The median accumulated dosage of RAI given was 80.7 (range 79.7-300.8) $\mathrm{mCi}$. A particular female patient who received RAI after prior isthmectomy and pyramidal lobe excision (considered to be hemithyroidectomy in this study) in September 2001 had a history of subtotal thyroid lobectomy for benign nodular goitre many years earlier at another hospital. Preoperative ultrasonography revealed only scant thyroid tissue on both lobes. The final histopathology confirmed a diagnosis of MIFTC with focal margin involvement. After discussions with the oncologist and patient, 


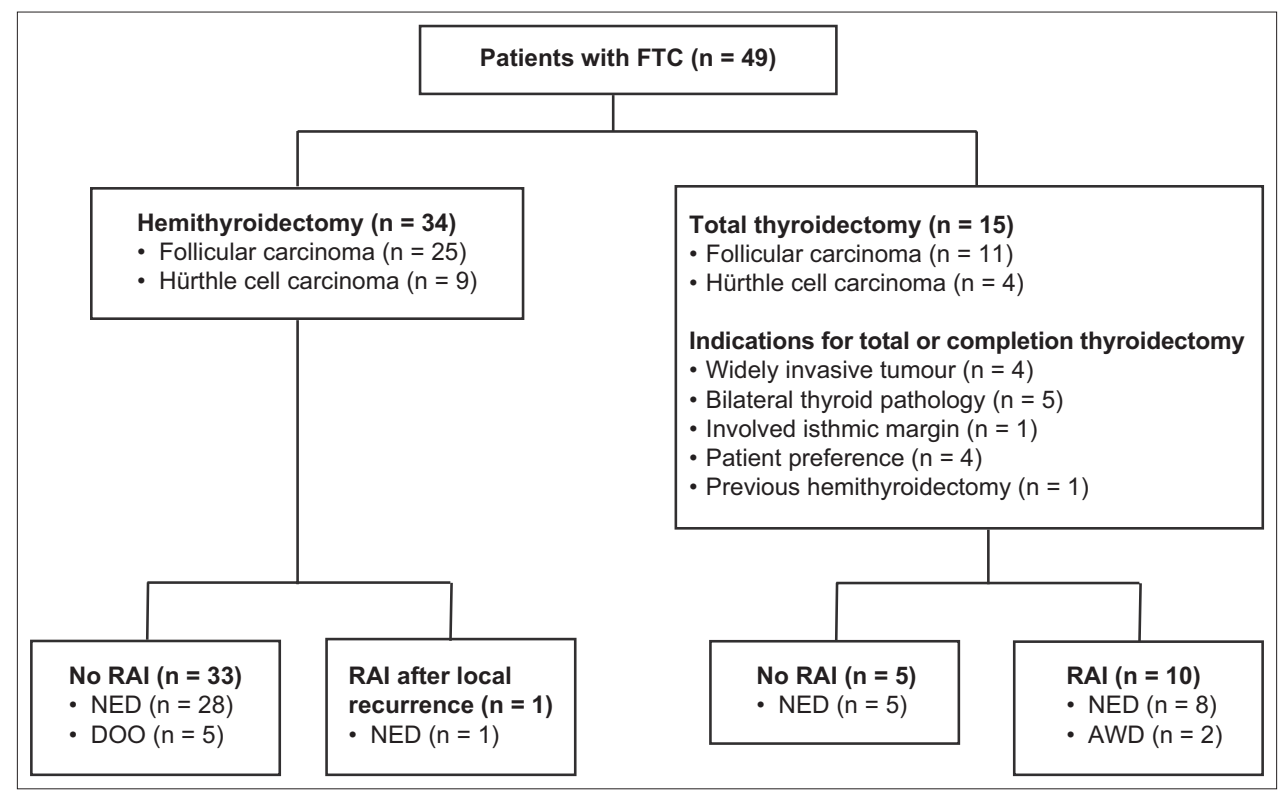

Fig. 1 Flowchart shows patient treatment and outcome. AWD: alive with disease; DOO: died of other illnesses; FTC: follicular carcinoma of the thyroid; NED: no evidence of disease; RAl: radioactive iodine

RAI ablation was provided in October 2001 without removal of the small thyroid remnants. However, she had local recurrence at the right thyroid lobe two years later. The recurrent tumour was then successfully salvaged by total thyroidectomy followed by further RAI. The patient showed no evidence of further disease relapse on follow-up over 13 years and was disease-free at the last follow-up in May 2014.

In the hemithyroidectomy group, no patient died of FTC, but there were five deaths due to other illnesses (Table II). Only one patient in the group had local recurrence. As described earlier, the tumour recurrence at the right thyroid lobe in this female patient was a remnant from an earlier isthmectomy and pyramidal lobe excision that was performed for MIFTC.

Of the 15 patients in the total thyroidectomy group, only four had WIFTC. The other 11 patients who had MIFTC underwent total or completion thyroidectomy instead of hemithyroidectomy for various reasons, such as bilateral thyroid lesions $(n=5)$, previous hemithyroidectomy for benign thyroid nodule $(n=1)$, isthmic margin involved by the tumour $(n=1)$ and patient preference $(n=4)$ (Fig. 1). None of these patients, except for two with WIFTC who were alive with disease at the last follow-up, had any evidence of disease (Table II). No patient from our cohort had died due to FTC at the time of the study.

The characteristics of the four patients with distant metastases and their extents are summarised in Table III. Our findings suggested that WIFTC was more likely to have distant metastases than MIFTC. However, no specific trend was noted with regard to the histopathology (follicular carcinoma vs. Hürthle cell carcinoma) and type of invasion (capsular carcinoma vs. vascular carcinoma).

The follow-up duration in the study was $86.9 \pm 56.6$ months. Due to the absence of disease-specific mortality in our small series, the Kaplan-Meier curve has not been shown. However, the five-, ten- and 15-year overall survival rates were 95\%, 91\% and $84 \%$, respectively (data not shown).
Table II. Treatment outcome at last follow-up.

\begin{tabular}{|lll|}
\hline Outcome & \multicolumn{2}{c|}{ No. (\%) } \\
\cline { 2 - 3 } & $\begin{array}{l}\text { Hemithyroidectomy } \\
(\mathbf{n}=\mathbf{3 4 )}\end{array}$ & $\begin{array}{l}\text { Total thyroidectomy } \\
(\mathbf{n}=\mathbf{1 5})\end{array}$ \\
\hline Local recurrence & $1(2.9)$ & $0(0)$ \\
\hline NED & $29(85.3)$ & $13(86.7)$ \\
\hline AWD & $0(0)$ & $2(13.3)$ \\
\hline DOO & $5^{*}(14.7)$ & $0(0)$ \\
\hline
\end{tabular}

*Died of lung cancer $(n=1)$, died of cholangitis $(n=1)$ or direct cause of death uncertain with senility (age $>90$ years) as a contributory cause $(n=3)$. AWD: alive with disease; DOO: died of other illnesses; NED: no evidence of disease

\section{DISCUSSION}

The debate on the optimal therapy for low-risk FTC is ongoing, and proponents for both hemithyroidectomy ${ }^{(4-6,11)}$ and total thyroidectomy ${ }^{(10,12)}$ coexist in the literature. According to the National Comprehensive Cancer Network treatment guidelines, observation and/or completion thyroidectomy are both legitimate for minimally invasive follicular or Hürthle cell carcinoma. ${ }^{(13)}$ More recently, the European Society of Endocrine Surgeons proposed an individualised approach, wherein hemithyroidectomy should be considered for patients with MIFTC satisfying the following criteria: (a) age $<45$ years; (b) absence of vascular invasion; (c) tumour size $<40 \mathrm{~mm}$; and (d) absence of nodal or distant metastases. In other cases, patients with MIFTC were to be treated with total thyroidectomy. ${ }^{(14)}$ Excellent disease control with hemithyroidectomy for MIFTC is attainable in highly specialised centres ${ }^{(5,6)}$ with abundant patient volume as well as experienced surgeons and pathologists. Indeed, a local study from a high-volume university centre, which reported its experience of managing 156 patients with FTC over 40 years, demonstrated no disease-specific mortality for MIFTC, and the authors recommended conservative surgery for MIFTC. ${ }^{(9)}$

In line with the results from other high-volume centres, the patient outcome in our series was excellent. When tumour 
Table III. Characteristics of patients with metastatic disease.

\begin{tabular}{|c|c|c|c|c|c|c|c|}
\hline $\begin{array}{l}\text { Age (yr)/ } \\
\text { gender }\end{array}$ & Tumour type & $\begin{array}{l}\text { Degree of } \\
\text { invasion }\end{array}$ & Invasion type & Operation & Site of metastasis & Outcome & $\begin{array}{l}\text { Duration of } \\
\text { follow-up (mth) }\end{array}$ \\
\hline $63 / F$ & $\begin{array}{l}\text { Hürthle cell } \\
\text { carcinoma }\end{array}$ & WIFTC & $\begin{array}{l}\text { Simultaneous } \\
\text { vascular and } \\
\text { capsular }\end{array}$ & Total thyroidectomy & $\begin{array}{l}\text { Skull, chest wall, left } \\
\text { scapula, right hip }\end{array}$ & AWD & 42 \\
\hline $57 / F$ & $\begin{array}{l}\text { Follicular } \\
\text { carcinoma }\end{array}$ & WIFTC & $\begin{array}{l}\text { Simultaneous } \\
\text { vascular and } \\
\text { capsular }\end{array}$ & Total thyroidectomy & $\begin{array}{l}\text { Bilateral lungs, skull, } \\
\text { sternum }\end{array}$ & AWD & 5 \\
\hline $32 / \mathrm{M}$ & $\begin{array}{l}\text { Follicular } \\
\text { carcinoma }\end{array}$ & WIFTC & Capsular & Total thyroidectomy & Bilateral lungs & NED & 38 \\
\hline $60 / F$ & $\begin{array}{l}\text { Follicular } \\
\text { carcinoma }\end{array}$ & MIFTC & Capsular & Total thyroidectomy & $\begin{array}{l}\text { Thoracic spine } \\
\text { (T5/6 vertebrae) }\end{array}$ & NED & 146 \\
\hline
\end{tabular}

AWD: alive with disease; F: female; FTC: follicular carcinoma of the thyroid; M: male; NED: no evidence of disease; MIFTC: minimally invasive FTC; WIFTC: widely invasive FTC

Table IV. Tumour recurrence and disease-specific mortality of FTC in previous studies in the literature.

\begin{tabular}{|c|c|c|c|c|c|c|}
\hline \multirow[t]{2}{*}{ Study } & \multirow[t]{2}{*}{ No. of patients } & \multirow{2}{*}{$\begin{array}{l}\text { Mean duration of } \\
\text { follow-up (yr) }\end{array}$} & \multirow[t]{2}{*}{ Details } & \multicolumn{3}{|c|}{$\%$} \\
\hline & & & & Overall & MIFTC & WIFTC \\
\hline \multirow[t]{3}{*}{ Huang et al(4) } & 234 & 9.0 & Recurrence & 26.0 & 0 & 42.1 \\
\hline & & & Distant metastasis & 17.5 & 0 & 28.3 \\
\hline & & & Cancer mortality & 17.5 & 0 & 28.3 \\
\hline \multirow[t]{2}{*}{ Lo et $\mathrm{al}^{(9)}$} & 156 & 14.4 & Recurrence & NA & NA & NA \\
\hline & & & Cancer mortality & 10.9 & 0 & 20.2 \\
\hline \multirow[t]{3}{*}{ Asari et $\mathrm{al}^{(10)}$} & 207 & NA & Recurrence & 11.1 & 5.5 & 20.0 \\
\hline & & & Distant metastasis & 18.4 & 9.4 & 32.5 \\
\hline & & & Cancer mortality & NA & 6.5 & 46.4 \\
\hline \multirow[t]{3}{*}{ Present study } & 49 & 7.2 & Recurrence & 2.0 & 2.2 & 0 \\
\hline & & & Distant metastasis & 8.2 & 2.2 & 75.0 \\
\hline & & & Cancer mortality & 0 & 0 & 0 \\
\hline
\end{tabular}

FTC: follicular carcinoma of the thyroid; MIFTC: minimally invasive FTC; NA: not available; WIFTC; widely invasive FTC

recurrence was considered, only 1 (2.9\%) patient from the hemithyroidectomy group ( $\mathrm{n}=34$ ) developed local relapse. Table IV summarises and compares our results of tumour recurrence, distant metastasis and cancer-related mortality with that of some other high-volume centres in the literature. Consistent with previous reports, MIFTC had a better prognosis than WIFTC, indicating that satisfactory treatment results in terms of disease-specific survival or tumour recurrence can be comparably achieved in low-volume hospitals as well. It is noteworthy that the occurrence of distant metastasis for WIFTC in our series was $75.0 \%$, which was much higher than the reported rates of up to $32.5 \%$ in the other series. A possible reason for this could be variations in the definition of WIFTC adopted by pathologists from different institutions. Other confounding factors for distant metastasis in our patients could also have accounted for the discrepancy; for instance, one patient had N1 disease at presentation, while another patient had major extrathyroid extension to the recurrent laryngeal nerve. It is also possible that the small sample size of our series might have contributed to some bias in the results.

Our results suggest a remarkable difference in the prognoses of MIFTC and WIFTC. Of the four patients with WIFTC, three were diagnosed with distant metastases at presentation. Furthermore, two of these four patients were alive with disease at the last follow-up. In contrast, all our patients with MIFTC were diseasefree at the last follow-up. This was similar to the findings of a population study in the United States that involved 1,200 patients with MIFTC and 4,208 patients with WIFTC, among whom only two patients with MIFTC died of disease-specific causes. ${ }^{(15)}$ The report suggested that overall survival was comparable to that of the general population, and total thyroidectomy and RAI were not associated with improved outcomes. The authors hence advocated thyroid lobectomy for patients with MIFTC. ${ }^{(15)}$ The prognosis of Hürthle cell carcinoma and follicular carcinoma was another dilemma. Patients with Hürthle cell carcinoma are more prone to develop nodal spread and less likely to take up RAI than patients with follicular carcinoma. ${ }^{(14)}$ Shaha et al have shown that Hürthle cell carcinoma was an adverse prognostic factor for FTC. ${ }^{(11)}$ Conversely, a population survey by the Surveillance, Epidemiology and End Results (SEER) Program of the National Cancer Institute, USA, from 1988 to 1993 demonstrated equivalent survival among patients with Hürthle cell carcinoma and follicular carcinoma. ${ }^{(16)}$ A more recent study on the SEER database found that the prognosis of Hürthle cell carcinoma had improved over the past four decades. ${ }^{(17)}$ Prior to 1979 , the survival rate among patients with Hürthle cell 
carcinoma was worse than that among follicular carcinoma patients. However, no difference was found in their survival for the period 1995-2004. The temporal change in the prognosis of Hürthle cell carcinoma might explain the conflicting results of past studies, ${ }^{(17)}$ as updated studies from Japan ${ }^{(18)}$ and Korea ${ }^{(19)}$ have demonstrated similar survival and tumour recurrence for these two types of carcinoma. Akin to these latest reports, Hürthle cell carcinoma did not portend an ominous prognosis when compared to follicular carcinoma in our study.

Distant metastases of FTC are not rare, as reported in our series and elsewhere. ${ }^{(3,4)}$ At times, distant metastasis might appear in patients with MIFTC. ${ }^{(12)}$ However, no patients with distant metastasis died due to FTC in our series, although two patients were alive with disease at the last follow-up and one patient had prolonged follow-up over 13 years. Therefore, aggressive treatment for FTC with M1 disease is advisable, as long-term survival with decent quality of life is possible for these patients. ${ }^{(20)} \mathrm{RAl}$ is beneficial ${ }^{(3,21)}$ and should be offered after total thyroidectomy. In contrast, there is no evidence to support the benefit of RAI for early or low-risk FTC. ${ }^{(22)}$ Likewise, for MIFTC that had metastasised, Goldstein et al ${ }^{(23)}$ failed to demonstrate any correlation between histology type (follicular carcinoma vs. Hürthle cell carcinoma) and degree of invasion (number of capsular or vascular invasion).

This study was not without weaknesses on account of inherent drawbacks such as its retrospective survey nature and small sample size. However, data was prospectively collected and the management policy remained consistent throughout the study period, which should minimise related biases. Also, as the mean follow-up duration was nearly 87 months, our findings could indicate meaningful long-term oncological outcomes for patients with FTC in spite of the small size of this series.

To sum up, satisfactory disease control and excellent survival for patients with MIFTC can be achieved by hemithyroidectomy even in community hospitals. Total thyroidectomy should be reserved for patients with WIFTC or aggressive tumours with nodal or distant metastasis.

\section{REFERENCES}

1. Davies L, Welch HG. Increasing incidence of thyroid cancer in the United States, 1973-2002. JAMA 2006; 295:2164-7.
2. Hedinger CE, Williams ED, Sobin LH. Histological typing of thyroid tumors. In: Hedinger CE, eds. International Histological Classification of Tumors. Berlin: Springer, 1988: 7-68.

3. Chow SM, Law SC, Mendenhall WM, et al. Follicular thyroid carcinoma: prognostic factors and the role of radioiodine. Cancer 2002; 95:488-98.

4. Huang CC, Hsueh C, Liu FH, Chao TC, Lin JD. Diagnostic and therapeutic strategies for minimally and widely invasive follicular thyroid carcinomas. Surg Oncol 2011; 20:1-6.

5. Thompson LD, Wieneke JA. Paal E, et al. A clinicopathologic study of minimally invasive follicular carcinoma of the thyroid gland with a review of the English literature. Cancer 2001; 91:505-24.

6. Nixon IJ, Ganly I, Patel SG, et al. Thyroid lobectomy for treatment of well differentiated intrathyroid malignancy. Surgery 2012; 151:571-9.

7. Chow TL, Choi CY, Lam SH. Disease control of differentiated thyroid carcinomas by hemithyroidectomy. Singapore Med J 2010; 51:311-4.

8. Kwan WY, Chow TL, Choi CY, Lam SH. Complication rates of central compartment dissection in papillary thyroid cancer. ANZ J Surg 2015; 85:274-8.

9. Lo CY, Chan WF, Lam KY, Wan KY. Follicular thyroid carcinoma: the role of histology and staging system in predicting survival. Ann Surg 2005; 242:708-15.

10. Asari R, Koperek O, Scheuba C, et al. Follicular thyroid carcinoma in an iodine-replete endemic goiter region: a prospectively collected, retrospectively analyzed clinical trial. Ann Surg 2009; 249:1023-31.

11. Shaha AR, Loree TR, Shah JP. Prognostic factors and risk group analysis in follicular carcinoma of the thyroid. Surgery 1995; 118:1131-6; discussion 1136-8.

12. Ban EJ, Andrabi A, Grodski S, et al. Follicular thyroid cancer: minimally invasive tumours can give rise to metastases. ANZ J Surg 2012; 82:136-9.

13. National Comprehensive Cancer Network. NCCN Clinical Practice Guidelines in Oncology: Thyroid Carcinoma Version 2.2013.

14. Dionigi G, Kraimps JL, Schmid KW, et al. Minimally invasive follicular thyroid cancer (MIFTC)--a consensus report of the European Society of Endocrine Surgeons (ESES). Langenbecks Arch Surg 2014; 399:165-84.

15. Goffredo P, Cheung K, Roman SA, Sosa JA. Can minimally invasive follicular thyroid cancer be approached as a benign lesion?: a population-level analysis of survival among 1,200 patients. Ann Surg Oncol 2013; 20:767-72.

16. Haigh PI, Urbach DR. The treatment and prognosis of Hürthle cell follicular thyroid carcinoma compared with its non-Hürthle cell counterpart. Surgery 2005; 138:1152-7; discussion 1157-8.

17. Nagar S, Aschebrook-Kilfoy B, Kaplan EL, Angelos P, Grogan RH. Hurthle cell carcinoma: an update on survival over the last 35 years. Surgery 2013; 154:1263-71; discussion 1271.

18. Sugino K, Kameyama K, Ito K, et al. Does Hürthle cell carcinoma of the thyroid have a poorer prognosis than ordinary follicular thyroid carcinoma? Ann Surg Oncol 2013; 20:2944-50.

19. Kim WG, Kim TY, Kim TH, et al. Follicular and Hurthle cell carcinoma of the thyroid in iodine-sufficient area: retrospective analysis of Korean multicenter data. Korean J Intern Med 2014; 29:325-33.

20. Shaha AR, Shah JP, Loree TR. Differentiated thyroid cancer presenting initially with distant metastasis. Am J Surg 1997; 174:474-6.

21. Pacini F, Schlumberger $M$, Harmer $C$, et al. Post-surgical use of radioiodine (131I) in patients with papillary and follicular thyroid cancer and the issue of remnant ablation: a consensus report. Eur J Endocrinol 2005; 153:651-9.

22. American Thyroid Association (ATA) Guidelines Taskforce on Thyroid Nodules and Differentiated Thyroid Cancer, Cooper DS, Doherty GM, et al. Revised American Thyroid Association management guidelines for patients with thyroid nodules and differentiated cancer. Thyroid 2009; 19:1167-214.

23. Goldstein NS, Czako P, Neill JS. Metastatic minimally invasive (encapsulated) follicular and Hurthle cell thyroid carcinoma: a study of 34 patients. Mod Pathol 2000; $13: 123-30$ 